\title{
Mathematical model of the pneumatic actuator follower system
}

\author{
Denis Medvedev*, Vyacheslav Grishhenko, Viktor Martynov, Evgeniy Ivliev, \\ Yurii Korol'kov \\ Don State Technical University, Rostov-on-Don, 344000, Russian Federation
}

\begin{abstract}
The article considers a method of controlling the motion of the output links of the tracking system of pneumatic actuators of technological equipment actuators. Dynamic and qualitative characteristics are improved by means of proportional-integral-differential (PID) controller. The mathematical model of actuator system, which includes power and control parts, has been developed. By calculation experiment the dynamic characteristics of the actuator have been obtained, from which it has been found possible to reduce the energy consumed by the actuator system to about $30 \%$.
\end{abstract}

\section{Introduction}

The modern level and further development of technological equipment is inextricably linked to the automation of production processes in companies and requires increased efficiency (energy consumption, speed, accuracy) of the actuator systems of technological equipment.

Pneumatic actuators and control systems are widely used in various industries, which is explained by high reliability of pneumatic systems, ease of operation, fire and explosion safety and low cost. At the same time, air can be used as a non-contact tool in technological operations and in control and measuring operations.

In combination with electronic control systems with the help of pneumatic systems it is possible to solve many problems, which were solved earlier by other means, much easier. However, in many cases the complexity of using pneumatic actuators to realize defined laws of motion casts doubt on their feasibility. Therefore, it is important already at the design stage to decide on the fundamental possibility and efficiency of using a pneumatic system $[1,2]$.

In this connection, the following tasks were set in the article: to develop a mathematical model of the pneumatic actuator follower system, a drive position cycle control algorithm and to find ways to improve the energy efficiency of the pneumatic actuator system.

\footnotetext{
* Corresponding author: 19medvedev97@mail.ru
} 


\section{Pneumatic actuator following system with proportional- integral-differential (PID) controller}

The combination of modern pneumatics and automation makes it possible to synchronize the velocity and position of the working mechanisms so precisely that high speeds, minimum operating times and precision of the parts can be realized in a single machine. But this, in turn, leads to higher requirements for drive dynamics and positioning accuracy of actuators.

Simulation of pneumatic system processes is complicated by non-stationarity of compressed gas flows [1-8]. Because of that, assumptions were made when forming the mathematical model of the actuator:

- The compressed air source characteristic is assumed constant $\mathrm{p}_{\mathrm{M}}$ - const, since the pressure line of the pneumatic actuator is connected to a receiver of sufficient volume through a pressure regulator;

- The thermodynamic process of gas state change is assumed to be adiabatic, as it proceeds in a short period of time;

- An ideal gas model is used in the description of pneumatic devices, since the pressure of compressed air is below 10 bar;

- Flow coefficients $\mu$ of control devices are assumed constant.

The mathematical model of the pneumatic actuator is developed on the basis of the accepted assumptions and in accordance with the calculation diagram in Figure 1 [2].

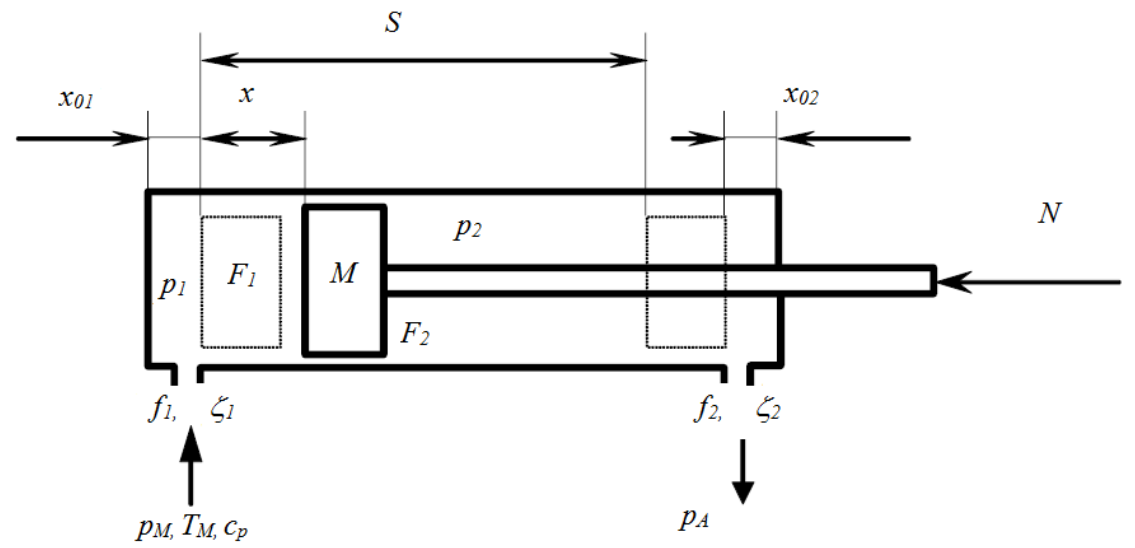

Fig. 1. Design diagram of a pneumatic actuator

\subsection{Mathematical model of the power part of the pneumatic actuator}

The equation of motion of the pneumatic actuator output link:

$$
M \frac{d^{2} x}{d t^{2}}=p_{1} F_{1}-p_{2} F_{2}-p_{\mathrm{A}}\left(F_{1}-F_{2}\right)-N
$$

where $P_{1}-$ pressure in the head end, $\mathrm{Pa} ; P_{2}-$ pressure in the rod end, $\mathrm{Pa} ; p_{A}-$ outlet pressure, $\mathrm{Pa} ; F$ - effective area of the piston, $\mathrm{m}^{2} ; N$ - static load, N.

Equation describing the change in pressure $P_{l}$ in the decreasing space: 


$$
\frac{d p_{1}}{d t}=\frac{k f_{1} \sqrt{R T_{M}}}{F_{1}\left(x+x_{01}\right) \sqrt{\varsigma_{1}}} \sqrt{p_{M}^{2}-p_{1}^{2}}-\frac{k p_{1}}{\left(x+x_{01}\right)} \frac{d x}{d t}
$$

where $T_{M}$ - absolute gas temperature, $\mathrm{K} ; R$ - gas constant, $\mathrm{J} /(\mathrm{kg} \mathrm{K}) ; k$ - adiabatic index (Poisson's coefficient); $f$ - pipe cross-section area, $\mathrm{m}^{2} ; x$ - piston position coordinate, $\mathrm{m}$; $x_{01}$ - reduced coordinate, characterizing the piston cavity harmful head end, $\mathrm{m} ; \varsigma-$ line resistance factor, valves; $p_{M}-$ supply pressure, $\mathrm{Pa}$.

Equation of gas pressure change in the outlet space:

$$
\frac{d p_{2}}{d t}=-\frac{k f_{2} \sqrt{R T_{M}}}{F_{2}\left(S-x+x_{02}\right) \sqrt{\varsigma_{1}}}\left(\frac{p_{2}}{p_{M}}\right)^{\frac{k-1}{2 k}} \sqrt{P_{2}^{2}-P_{\mathrm{A}}^{2}}+\frac{k p_{2}}{\left(S-x+x_{02}\right)} \frac{d x}{d t},
$$

where $S$ - maximum piston stroke, $\mathrm{m} ; x_{02}$ - reduced coordinate characterizing the volume of the harmful space of the rod cavity, $\mathrm{m}$.

The coordinate of the piston position $\mathrm{x}$ varies between 0 and $S$, i.e. the piston in the pneumatic actuator moves from stop to stop. This limitation must be reflected in the mathematical model. If the pneumatic actuator is in the leftmost position, then it is necessary to artificially set acceleration, velocity of motion and coordinate equal to zero in the program immediately after acceleration calculation, i.e. the condition should be added to the obtained equations of the mathematical model of the pneumatic actuator:

$$
\text { if } x<0 \text {, то } \frac{d^{2} x}{d t^{2}}=\frac{d x}{d t}=x=0 \text {. }
$$

Similarly, for the case where the piston reaches the end at the rightmost position, we must write a condition limiting the coordinate $\mathrm{x}$ to the length of the actuator stroke $S$ :

$$
\text { if } x \geq S, \text { то } \frac{d^{2} x}{d t^{2}}=\frac{d x}{d t}=x=0
$$

The final mathematical model of a typical pneumatic actuator is as follows:

$$
\left.\begin{array}{c}
M \frac{d^{2} x}{d t^{2}}=p_{1} F_{1}-p_{2} F_{2}-p_{\mathrm{A}}\left(F_{1}-F_{2}\right)-N, \\
\frac{d p_{1}}{d t}=\frac{k f_{1} \sqrt{R T_{M}}}{F_{1}\left(x+x_{01}\right) \sqrt{\varsigma_{1}}} \sqrt{p_{M}^{2}-p_{1}^{2}}-\frac{k p_{1}}{\left(x+x_{01}\right)} \frac{d x}{d t}, \\
\frac{d p_{2}}{d t}=-\frac{k f_{2} \sqrt{R T_{M}}}{F_{2}\left(S-x+x_{02}\right) \sqrt{\varsigma_{1}}}\left(\frac{p_{2}}{p_{M}}\right)^{\frac{k-1}{2 k}} \sqrt{P_{2}^{2}-P_{\mathrm{A}}^{2}}+\frac{k p_{2}}{\left(S-x+x_{02}\right)} \frac{d x}{d t}, \\
\text { if } x<0, \text { то } \frac{d^{2} x}{d t^{2}}=\frac{d x}{d t}=x=0, \\
\text { if } x \geq S \text {, то } \frac{d^{2} x}{d t^{2}}=\frac{d x}{d t}=x=0 .
\end{array}\right\}
$$

\subsection{Equation of the control part of a pneumatic actuator (PID controller}

This is the most common regulator structure in process control and servomechanisms (Figure 2) [4]. 


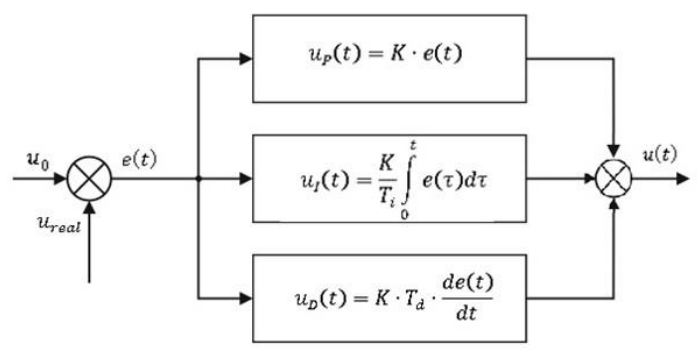

Fig. 2. PID controller structure

The PID controller produces an output signal that is the sum of the three components of proportional control, integral control and derivative control. The first part $u_{P}(t)$ is proportional to the error of the output quantity $e(t)$, i.e. the difference between the output quantity and the reference value, the second part $u_{I}(t)-$ is integral in time of the error of the output quantity, and the third part $u_{D}(t)-$ is derivative of the error. The equation of the classical PID controller is [4-19]:

$$
u(t)=u_{0}+K\left[e(t)+\frac{1}{T_{i}} \int_{0}^{t} e(\tau) d \tau+T_{d} \frac{d e(t)}{d t}\right]=u_{0}+u_{P}(t)+u_{I}(t)+u_{D}(t),
$$

where $K$ - the controller gain; $T_{i}$ - the integration time constant, s; $T_{d}$ - the differentiation time constant, $\mathrm{s} ; u_{0}-$ the correction value or offset that adjusts the average output level of the controller.

\subsection{Simulation experiment}

The solution of the resulting model was performed on a computer using the Simulink graphical simulation environment based on the Matlab program (Figure 3). Reliability of the computational experiment is confirmed by solving the system of equations by the ode $23 \mathrm{t}$ method, it is an implicit trapezoidal method with interpolation. This method gives good results when solving problems describing oscillating systems with almost harmonic output signal. With moderately rigid systems of differential equations, it can give high solution accuracy.

To conduct a full-scale experiment, as well as to assess the influence of various factors on the speed and accuracy of positioning, the bench equipment was developed (Figure 4), which allows to conduct a multifactorial experiment. To collect and process the information, an automated measuring complex was developed, which essentially includes a personal computer, a programmable logic controller and sensors (Figure 4).

As a result of dynamic calculation of the pneumatic actuator (Figure 1) we obtain time characteristics, which allow evaluating stability and quality of the transient process. 


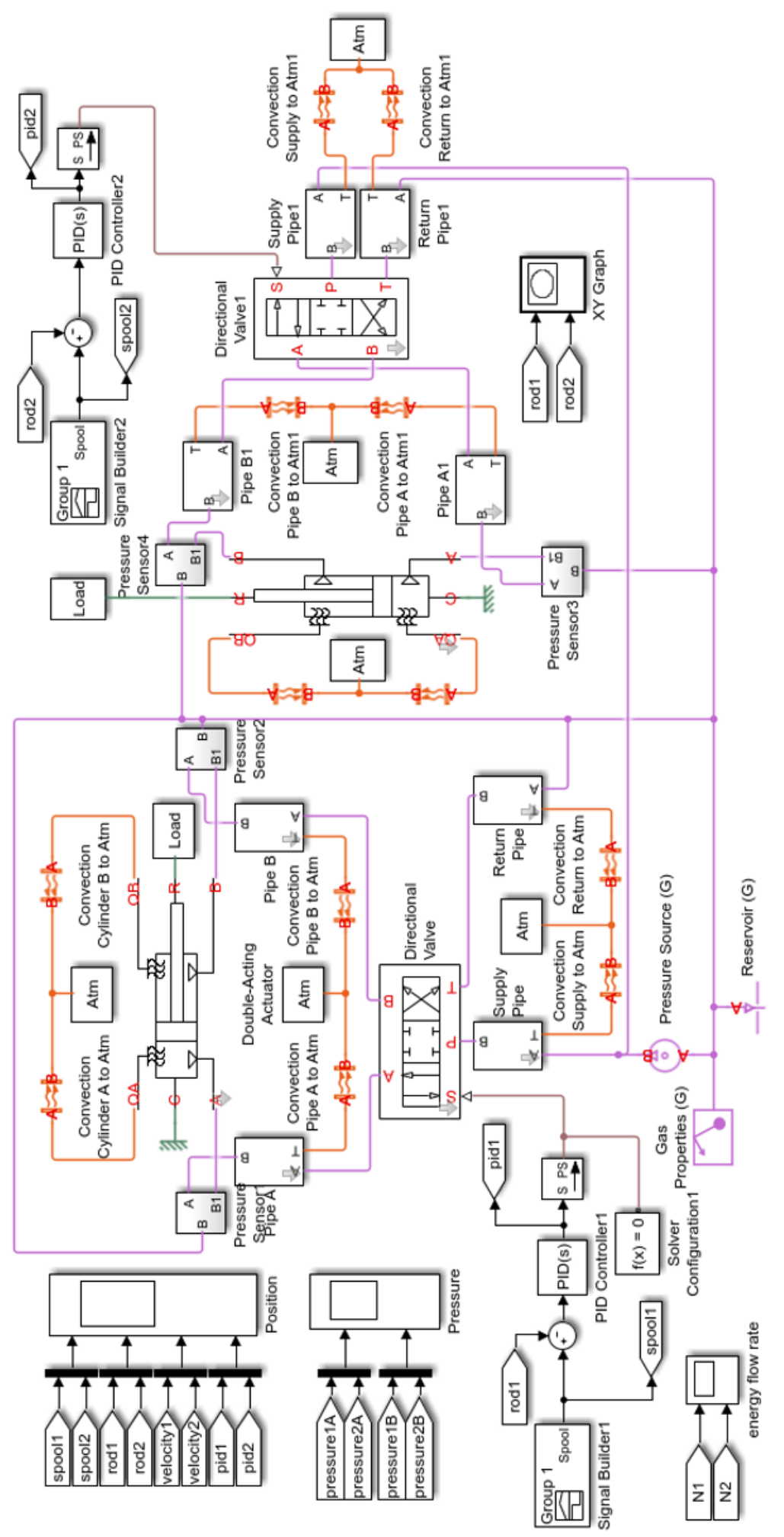

Fig. 3. Mathematical model 


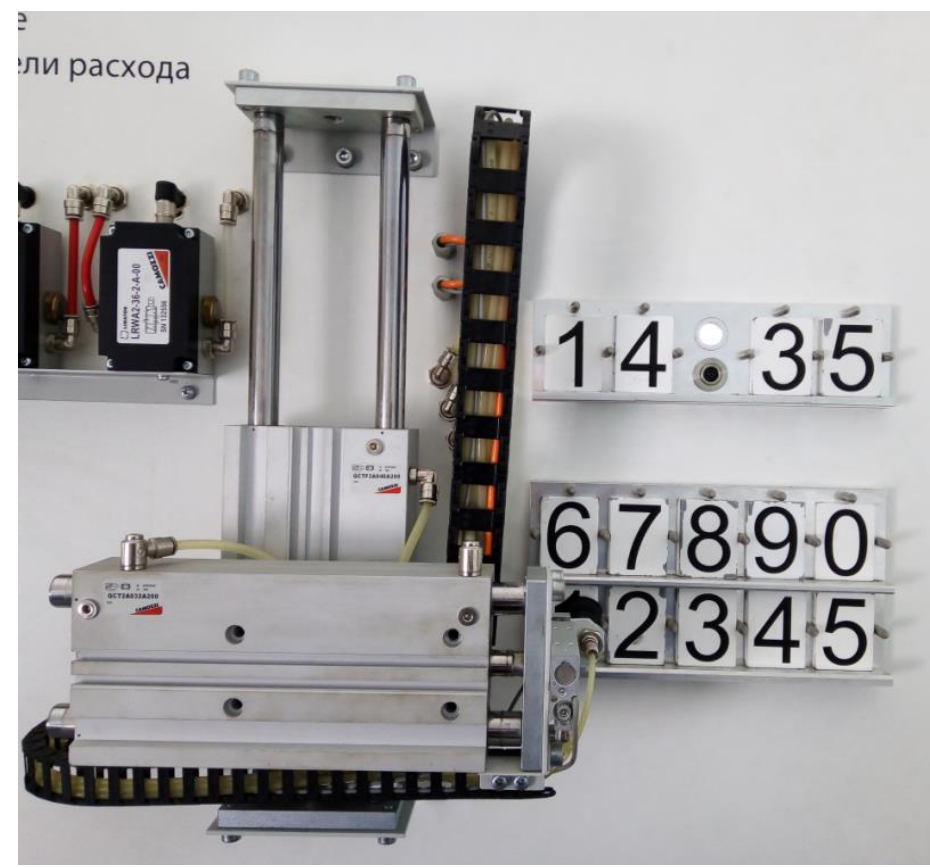

Fig. 4. Stand for the study of the pneumatic actuator

The results of dynamic calculation are presented in the form of dependences of the coordinate, velocity of movement (Figure 5) and air pressure on time (Figure 6). At the first second, a signal comes to the regulator and after 0.3 seconds the rod of the first (horizontal) cylinder is set on the value of $0.1 \mathrm{~m}$. The second (vertical) cylinder piston rod is set to $0.15 \mathrm{~m}$ after $0.7 \mathrm{~s}$ (Figure 7 ).

At 3 seconds, the signal to the regulator is reset to 0 and the rods of both cylinders return to their original positions (Figure 5).

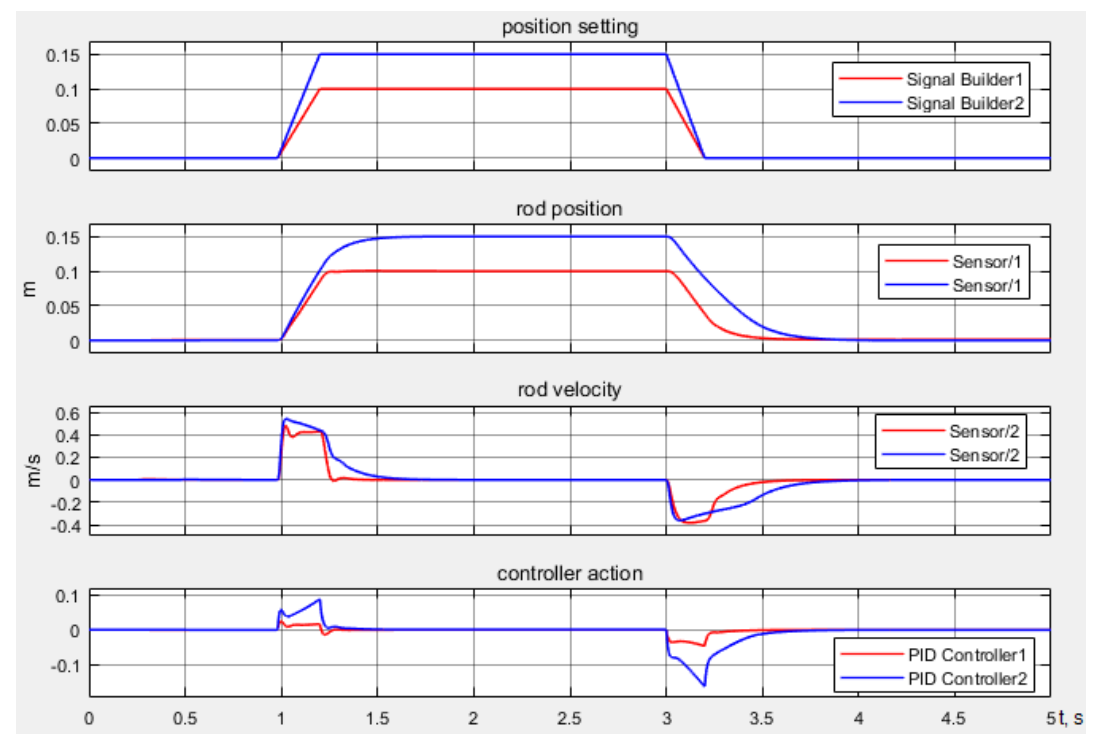

Fig. 5. Dependence of velocity and rod movement on time 

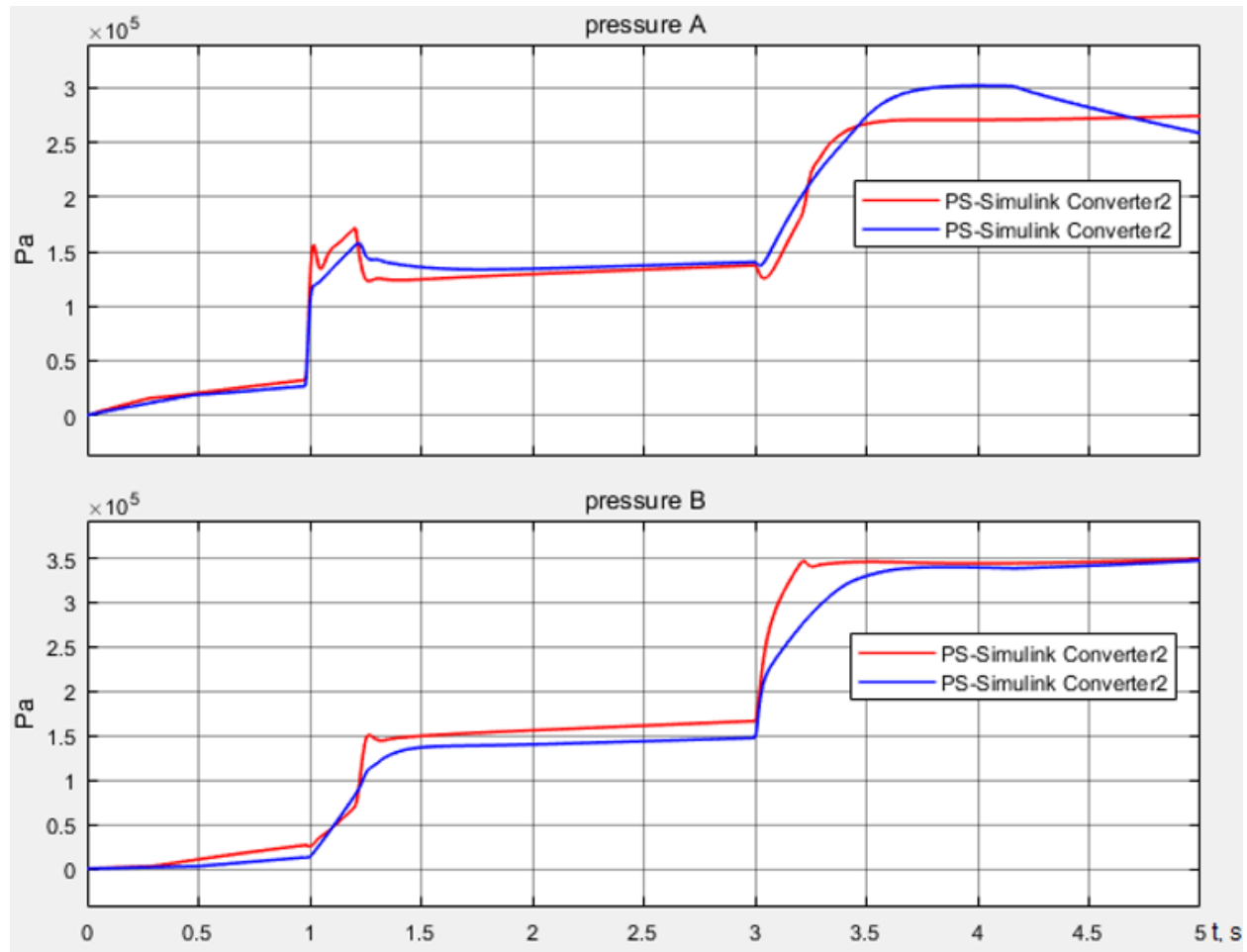

Fig. 6. Dependence of pressure in the piston (A) and rod (B) cavity of the cylinder on time

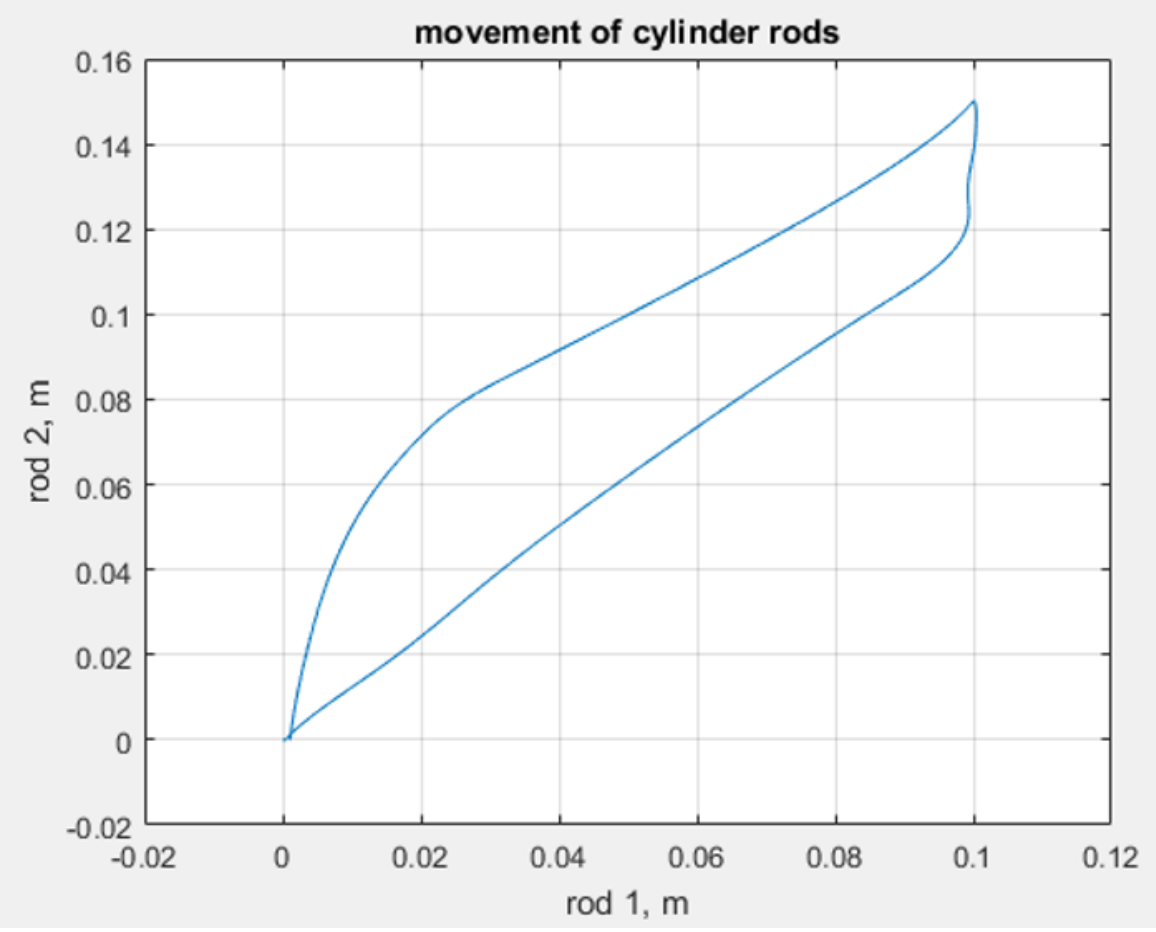

Fig. 7. Movement of two rods in space 
The quality of the transients is confirmed by the dependencies obtained in the graphs (Figure 5,6). The majority of transients are aperiodic. The process of extending of the horizontally located pneumatic cylinder is oscillatory (Figure 5). Oscillations of the rod displacement velocity are damped, the damping decrement is equal to 1.13 , the oscillation frequency is $10.75 \mathrm{~Hz}$ and the setting time is $0.42 \mathrm{~s}$.

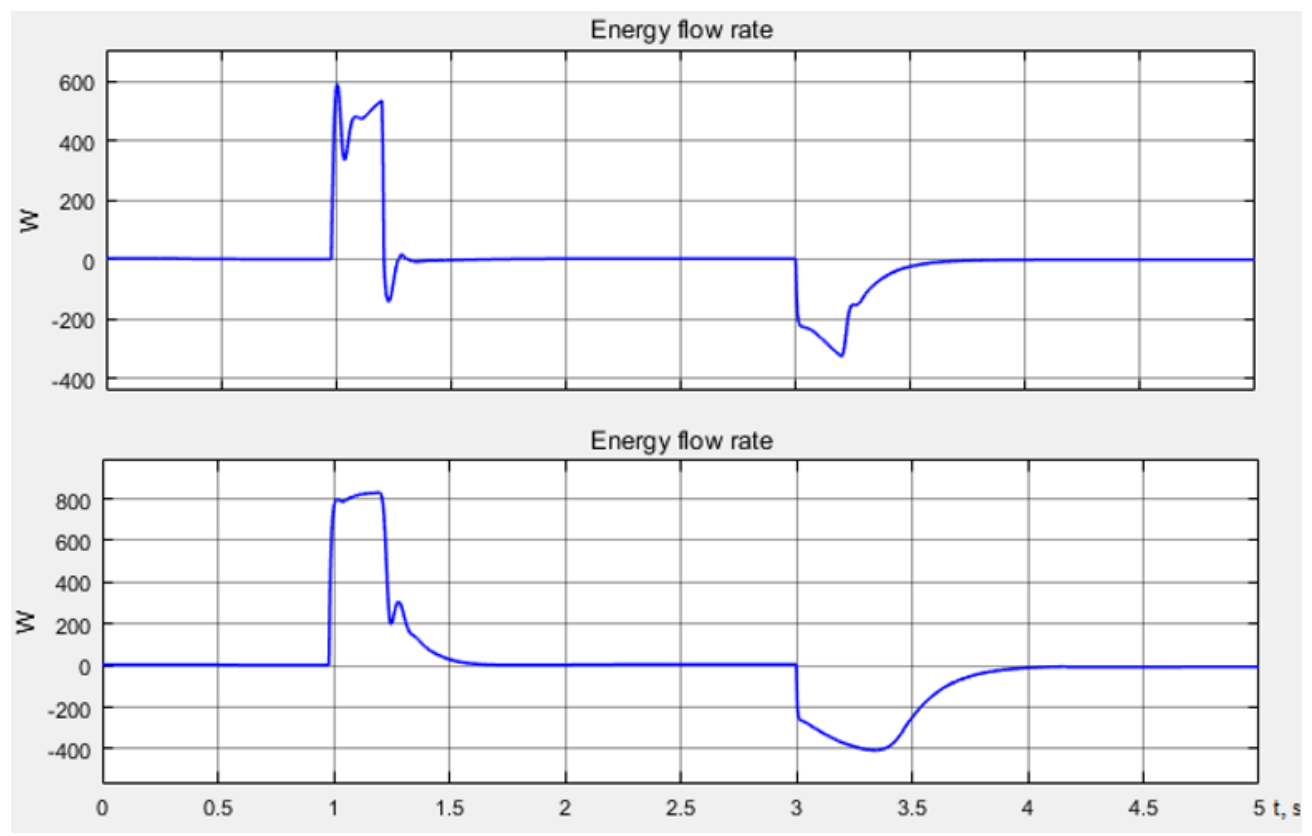

Fig. 8. Energy flow rate

As can be seen from the graph (Figure 6), extending the cylinder rod requires half the pressure, relative to the system pressure. At the same time, the energy consumption in the piston cavity of the first (horizontal) cylinder at the peak is $600 \mathrm{~W}$, the second (vertical) is $800 \mathrm{~W}$ (Figure 8). In addition, it can be seen from the graph (Figure 6) that the PID controller starts the reversal process by applying a control signal to open the distributor's flow channels. This leads to an increase in pressure in the pressure chamber of the cylinder. However, there is already air remaining in the cylinder under pressure which can be used to reduce the energy input for the return of the piston. This requires the development of a more optimal actuator system control law, which will be the target of future work.

\section{Conclusion}

As a result, a mathematical model of pneumatic actuator has been developed, which allows conducting dynamic analysis at the stages of development, which significantly reduces the time and money for prototype development and improves the quality of dynamic processes of technological equipment mechanisms. The possibility of drive performance increase with the use of PID controller is shown. As a result, the positioning process time is reduced, resulting in shorter cycle times and consequently higher productivity. A calculation experiment has shown the dynamic behaviour of the actuator, which can reduce the energy consumption of the actuator system by up to $30 \%$. 


\section{References}

1. Grishchenko V.I. Increase in accuracy of the high-speed pneumohydraulic drive of mechanisms of machine. PhD dissertation, (2010)

2. Donskoy A.S. Mathematical modeling of processes in pneumatic drives: Textbook. Benefit, (2009)

3. Sirotenko A.N., Grishchenko V.I., Partko S.A., Kilina M.S. IOP Conference Series: Materials Science and Engineering 1029 (2020) https://doi.org/10.1088/1757899X/1029/1/012087.

4. Dao T.A., Sidorenko V.S., Dymochkin D.D. Vestnik of Don State Technical University, 15(4): 46-53 (2015) https://doi.org/10.12737/16077

5. Harris P., O’Donnell G.E., Whelan T. Leveraging Technology for a Sustainable World (2012), https://doi.org/10.1007/978-3-642-29069-5_62

6. Ilyukhin Yu., Kharchenko A., Arfikyan S. Vestnik of Don State Technical University 10(8): 1184-1189 (2010)

7. Yang, F.; Tadano, K.; Li, G.; Kagawa, T.; Peng, J. Inf. Sci. 645, 143-153 (2016)

8. J. Hepke, J. Weber. Energy saving measures on pneumatic drive systems. Proceedings from the 13th Scandinavian International Conference on Fluid Power SICFP (2013). doi: 10.3384/ecp1392a47

9. Antypas IR, Dyachenko AG, Sirotenko AN, Partko SA and Savostina TP. IOP Conference Series: Earth and Environmental Science, 403(1), 012005, (2019)

10. R. Saidur, N.A. Rahim, M. Hasanuzzaman. Renewable and Sustainable Energy Reviews, 14 (4). 1135-1153 (2010)

11. Yang, F.; Tadano, K.; Li, G.; Kagawa, T.; Peng, J. Inf. Sci., 645, 143-153. (2016)

12. Elvira Rakova and Jürgen Weber. Procedia Engineering, 106, 149 - 157, (2015)

13. Blagojevic, V.; Seslija, D.; Dudic, S.; Randjelovic, S. Energies, 13, 3711. (2020)

14. Krytikov G, Strizhak M and Strizhak V Eastern-European Journal of Enterprise Technologies, 7(85), 38-44 (2017)

15. D.D. Medvedev, V.I. Timofeev, A.A. Zotov. Modern informatization problems in simulation and social technologies: Proc. Of the XXV-th International Open Science Conference. Yelm, WA, USA: Science Book Publishing House, (2020)

16. Kuzmenko A.A., Sinitsyn A.S. Vestnik of Don State Technical University, 14(1): 154161. (2014) https://doi.org/10.12737/3514

17. Antonenko V.I. Vestnik of Don State Technical University, 14(4): 158-165, (2014) https://doi.org/10.12737/6895

18. Aydinyan A.R., Tsvetkova O.L., Molchanov A.A. Vestnik of Don State Technical University, 14(3): 88-95 (2014) https://doi.org/10.12737/5713

19. Karnaukhov N.F., Smyatsky D.A., Filimonov M.N., Rudnev K.I. Vestnik of Don State Technical University 19(4): 342-348, (2019) https://doi.org/10.23947/1992-59802019-19-4-342-348

20. Tugengold A.K., Izyumov A.I., Voloshin R.N., Solomykin M.Y. Vestnik of Don State Technical University, 17(2): 70-78 (2017) https://doi.org/10.23947/1992-5980-201717-2-70-78 